\title{
Identification and characterization of histone deacetylases in tomato (Solanum Iycopersicum)
}

\author{
Linmao Zhao ${ }^{1,2+}$, Jingxia Lu ${ }^{1,2+}$, Jianxia Zhang ${ }^{1,3}$, Pei-Ying $\mathrm{Wu}^{3}$, Songguang Yang ${ }^{1 *}$ and Keqiang $\mathrm{Wu}^{3 *}$ \\ ${ }^{1}$ Key Laboratory of Plant Resources Conservation and Sustainable Utilization, South China Botanical Garden, Chinese Academy of Sciences, Guangzhou, China \\ ${ }^{2}$ College of Life Sciences, University of Chinese Academy of Sciences, Beijing, China \\ 3 Institute of Plant Biology, National Taiwan University, Taipei, Taiwan
}

\section{Edited by:}

Mario Alberto Arteaga-Vazquez,

Universidad Veracruzana, Mexico

Reviewed by:

Zhong Chen, National Institute of

Education/Nanyang Technological University, Singapore

Shaomin Bian, Jilin University, China

\section{*Correspondence:}

Songguang Yang, Key Laboratory of Plant Resources Conservation and Sustainable Utilization, South China Botanical Garden, Chinese Academy of Sciences, Xingke Road 723 ,

Tianhe District, Guangzhou 510650,

China

e-mail: yangsongguang@scbg.ac.cn;

Keqiang Wu, Institute of Plant

Biology, National Taiwan University,

Taipei 106, No. 1, Sec. 4, Roosevelt

Road, 10617 Taipei, Taiwan

e-mail: kewu@ntu.edu.tw

${ }^{\dagger}$ These authors have contributed

equally to this work.
Histone acetylation and deacetylation at the $\mathrm{N}$-terminus of histone tails play crucial roles in the regulation of eukaryotic gene activity. Histone acetylation and deacetylation are catalyzed by histone acetyltransferases and histone deacetylases (HDACs), respectively. A growing number of studies have demonstrated the importance of histone deacetylation/acetylation on genome stability, transcriptional regulation, development and response to stress in Arabidopsis. However, the biological functions of HDACs in tomato have not been investigated previously. Fifteen HDACs identified from tomato (Solanum lycopersicum) can be grouped into RPD3/HDA1, SIR2 and HD2 families based on phylogenetic analysis. Meanwhile, 10 members of the RPD3/HDA1 family can be further subdivided into four groups, namely Class I, Class II, Class III, and Class IV. High similarities of protein sequences and conserved domains were identified among SIHDACs and their homologs in Arabidopsis. Most SIHDACs were expressed in all tissues examined with different transcript abundance. Transient expression in Arabidopsis protoplasts showed that SIHDA8, SIHDA1, SIHDA5, SISRT1 and members of the HD2 family were localized to the nucleus, whereas SIHDA3 and SIHDA4 were localized in both the cytoplasm and nucleus. The difference in the expression patterns and subcellular localization of SIHDACs suggest that they may play distinct functions in tomato. Furthermore, we found that three members of the RPD3/HDA1 family, SIHDA1, SIHDA3 and SIHDA4, interacted with TAG1 (TOMATO AGAMOUS1) and TM29 (TOMATO MADS BOX29), two MADS-box proteins associated with tomato reproductive development, indicating that these HDACs may be involved in gene regulation in reproductive development.

Keywords: histone deacetylases, subcellular localization, gene expression, MADS-box proteins, tomato

\section{INTRODUCTION}

In eukaryote cells, genetic information encoded by DNA is packed extensively forming the chromatin structure. The fundamental unit of chromatin is a nucleosome containing a histone octamer (two copies of histone $\mathrm{H} 2 \mathrm{~A}, \mathrm{H} 2 \mathrm{~B}, \mathrm{H} 3$, and $\mathrm{H} 4$ ) wrapped on approximately 147 base pairs of DNA (Luger et al., 1997). Each histone has a structured globular domain and an unstructured amino-terminal tail that extends from the core nucleosome (Campos and Reinberg, 2009). The N-terminal tails of histone proteins provide sites for a variety of post-translational modifications, such as acetylation, phosphorylation, methylation, glycosylation, ubiquitation and ADP-ribosylation [3]. Among these modifications, histone acetylation is one of the well characterized

\footnotetext{
Abbreviations: bp, base pair; BiFC, bimolecular fluorescence complementation; cDNA, complementary DNA; dpa, days post anthesis; GST, glutathione S-transferase; HATs, histone acetyltransferases; HDACs, histone deacetylases; MADS-box, Minichromosome Maintenance 1, Agamous, Deficiens and human serum response factor SRF; MG, mature green; PCR, polymerase chain reaction; RR, red ripe; SEP, SEPELATTA; TAG1, TOMATO AGAMOUS11; TAGL11, TOMATO AGAMOUS-LIKE1; TM4, TOMATO MADS BOX4; TM29, TOMATO MADS BOX29; YFP, yellow fluorescence proteins.
}

post-translational modifications (Allfrey et al., 1964; Lusser et al., 2001).

The acetylation state of the $\varepsilon$-amino group of conserved lysine residues within all four core histones was regulated by the opposing activities of histone acetyltransferases (Brownell et al., 1996) and histone deacetylases (HDACs) (Nagy et al., 1997). HDACs can remove acetyl groups from histone and non-histone substrates including transcription factors and other proteins involved in DNA repair and replication, metabolism, cytoskeleton dynamics, apoptosis and cell signaling (Yang and Seto, 2007). Based on sequence similarity and cofactor dependency, HDACs in all eukaryotes are divided into three families: RPD3/HDA1 (Reduced Potassium Dependence 3/Histone Deacetylase 1), SIR2 (Silent Information Regulator 2), and plantspecific HD2 (Histone Deacetylase 2) (Pandey et al., 2002; Yang and Seto, 2007). Members of the SIR2 family (sirtuins) have a catalytic domain that is characterized by the requirement for nicotine adenine dinucleotide (NAD) as a cofactor (Haigis and Guarente, 2006), while members of the RPD3/HDA1 family share sequence homology in the HDAC domain and require the $\mathrm{Zn}^{2+}$ cofactor for deacetylase activity (Yang and Seto, 2007). 
In the past decade, plant HDACs have drawn considerable research attention and an increasing number of HDACs were purified, identified and characterized from plants such as maize, Arabidopsis, rice, barley (Demetriou et al., 2009), potato (Lagace et al., 2003), grape (Busconi et al., 2009), tobacco (Bourque et al., 2011), and tomato (Cigliano et al., 2013b). The Arabidopsis genome encodes 18 HDACs. Twelve of them belong to the RPD3/HDA1 superfamily, two are the SIR2 family and four are members of the plant specific HD2 family (Pandey et al., 2002; Alinsug et al., 2009). The Arabidopsis HDACs play a vital role in regulating gene expression in various biological processes. For instance, HDA6 was involved in transgene silencing and maintain of DNA methylation (Aufsatz et al., 2002; Probst et al., 2004). While HDA19, the closest homolog of HDA6, was shown to be important for proper vegetative development as hda19 mutants displayed various developmental abnormalities (Tian and Chen, 2001; Long et al., 2006; Zhou et al., 2013). HDA18 is required for the cellular patterning in the root epidermis (Xu et al., 2005; Liu et al., 2013a), whereas HDA7 is crucial for female gametophyte development and embryogenesis in Arabidopsis (Cigliano et al., 2013a). Furthermore, HDA14 is an $\alpha$-tubulin decetylase associated with $\alpha / \beta$-tubulin and enriched in microtubule fractions by direct association with the PPP-type phosphatases PP2A (Tran et al., 2012). Silencing of HD2A in Arabidopsis resulted in aborted seed development (Wu et al., 2000), while overexpression of $H D 2 A$ caused morphological defects of leaves and flowers, delayed flowering and aborted seed development (Zhou et al., 2004). In addition, HD2A and HD2B were found to act independently with ASYMMETRIC LEAVES1 (AS1) and AS2 to control miR165/166 distribution and the development of adaxialabaxial leaf polarity (Ueno et al., 2007). More recently, the SRT2 in Arabidopsis was showed to be predominantly localized at the inner mitochondrial membrane and to interact with a small number of protein complexes mainly involved in energy metabolism and metabolite transport (Konig et al., 2014).

The molecular mechanisms of fruit development are not well-understood. Genetic studies in Arabidopsis have uncovered the genetic network that patterns the Arabidopsis fruit. For instance, the members of MADS-box transcription factor family, FRUITFULL (FUL) and SHATTERPROOF 1/2 (SHP 1/2), are necessary for proper valve development ( $\mathrm{Gu}$ et al., 1998; Ferrandiz et al., 2000). Furthermore, INDEHISCENT (IND) and ALCATRAZ (ALC), two bHLH transcription factors, are both necessary for the differentiation of the dehiscence zone between the valve and replum regions (Girin et al., 2011; Groszmann et al., 2011). Previous data also showed that HDACs were involved in fruit development. For instance, AtHDA19 mutations induce embryonic defects and seed set reduction (Tian et al., 2003). Silencing and overexpression of AtHD2A both severely affect seed development (Wu et al., 2000; Zhou et al., 2004). In addition, hda6 mutants were reported to exhibit reduced fertility (Aufsatz et al., 2002), while mutations of AtHDA7 led to partial failure of ovule/female gametophyte development and seed abortion (Cigliano et al., 2013a).

Compared to Arabidopsis, relatively few HDACs were characterized in other plant species. In this study, 15 HDACs were identified in the tomato genome. The expression patterns and subcellular localization of tomato HDACs (SlHDACs) were investigated. Furthermore, the interaction between SIHDACs and the MADS-box proteins involved in reproductive development sheds light on potential functions of SIHDACs during reproductive development.

\section{MATERIALS AND METHODS PLANT MATERIAL AND GROWTH CONDITIONS}

Solanum lycopersicum cultivar Henz1706 was kindly provided by Wang Ying (South China Botanical Garden). Plants were grown in soil in a controlled-environment greenhouse with a long photoperiod ( $16 \mathrm{~h} \mathrm{light} / 8 \mathrm{~h}$ dark) at $23 \pm 1^{\circ} \mathrm{C}$. The tomato seeds were accelerated germination by putting the seeds in a $28^{\circ} \mathrm{C}$ incubator for 2 days before sowing.

\section{IDENTIFICATION OF SLHDAC GENES}

The HDACs sequences of Arabidopsis thaliana obtained in the TAIR database (http://www.arabidopsis.org/) were used to perform a search in the Solamum lycopersicum genome using the BLASTP program in the SGN browser (http://solgenomics.net/ tools/blast/index.pl). All the sequences acquired were removed the duplicates and analyzed for the recognizable domains using BLAST-based NCBI conserved domain searches (http://www. ncbi.nlm.nih.gov/Structure/lexington/lexington.cgi). The protein sequences were further verified using the HMMER-based SMAT Website (http://smart.embl-heidelberg.de/) and the Pfam program (http://pfam.janelia.org/). The domain architecture was drawn using DOG2.0 software (Ren et al., 2009).

\section{PHYLOGENETIC CONSTRUCTION}

The tomato HDAC proteins identified in this work along with the proteins from Arabidopsis were aligned with ClustalX. The phylogenetic analysis was performed using the MEGA3.0 program (Kumar et al., 2004). Neighbor-Joining method and the Poisson correction were used to infer the evolutionary history and compute the evolutionary distances. In addition, all positions containing alignment gaps and missing data were eliminated only in pairwise sequence comparisons.

\section{QUANTITATIVE REAL-TIME REVERSE TRANSCRIPTION-PCR (qRT-PCR) ASSAYS}

Total RNA was extracted with Trizol reagent (Invitrogen) according to the manufacturer's protocol. The cDNAs were synthesized from $2 \mu \mathrm{g}$ total RNA using the TransScript ${ }^{\mathrm{TM}}$ One-Step gDNA Removal and cDNA Synthesis Supermix kit (TransGen Biotech). Real-Time PCR was performed with $\mathrm{iTaq}^{\mathrm{TM}}$ Universal SYBR ${ }^{\circledR}$ Green Supermix (BIO-RAD) using ABI 7500 Fast Real-Time PCR system. The gene-specific primers for real-time PCR were designed by primer 3.0 (Untergasser et al., 2012) and listed in Supplementary Table 1. Tomato Actin (Solyc03g078400) served as an internal control.

\section{SUBCELLULAR LOCALIZATION ASSAYS}

The full length cDNAs of SlHDACs were subcloned into the pSAT6-EYFP_N1 vector (Tzfira et al., 2005) to create the SIHDAC-YFP constructs. The protoplast isolation and transient expression were conducted as described previously (Yoo 
et al., 2007). Mesophyll protoplasts were isolated using the wellexpanded leaves from 3-week-old Arabidopsis Col-0 plants. The SIHDAC-YFP fusion plasmid and the nuclear maker VirD2-NLS mCherry were co-transfected into protoplasts $\left(3 \times 10^{4}\right.$ protoplasts) using the PEG-calcium solution $(0.4 \mathrm{~g} / \mathrm{mL}$ PEG 4000, $0.2 \mathrm{M}$ mannitol, $\left.0.1 \mathrm{M} \mathrm{CaCl}_{2}\right)$. After washed and resuspended with W5 solution ( $154 \mathrm{mM} \mathrm{Nacl}, 125 \mathrm{mM} \mathrm{CaCl}_{2}, 5 \mathrm{mM} \mathrm{KCl}$, $5 \mathrm{mM}$ glucose, $2 \mathrm{mM}$ MES), mesophyll protoplasts were incubated under white light for 12-18 h. The YFP fluorescence was examined and imaged with a confocal microscope.

\section{YEAST TWO-HYBRID, BiFC AND PULL-DOWN ASSAYS}

Yeast two-hybrid assays were performed using the Matchmaker ${ }^{\mathrm{TM}}$ Gold Yeast Two-Hybrid Systems (Clontech). Constructs were generated by cloning SIHDACs into pGADT7 vectors and four selected MADS-box genes into pGBKT7 vectors. Different pairs of bait and prey constructs were co-transformed into yeast strain Gold by the lithium acetate method, and yeast cells were grown on minimal medium/-Leu-Trp according to the manufacturer's instructions. Transformed colonies were plated onto minimal medium/-Leu/-Trp/-Ade/-His and minimal medium/-Leu/-Trp/Ade/-His plates containing $4 \mathrm{mg} / \mathrm{mL} \mathrm{X- \alpha -Gal}$.

For BiFC assay, the cDNAs of SIHDA1, SIHDA2, SIHDA4, TAG1, and TM29 were cloned into serial pSAT1 vectors (Tzfira et al., 2005) containing either amino- or carboxyl terminal Enhanced Yellow Fluorescence Protein (EYFP) fragments by In-Fusion cloning (Clontech, In-Fusion ${ }^{\circledR} \mathrm{HD}$ Cloning Kit, Cat\#639650). Plasmids of the corresponding N- and C-terminal fusions of YFP were cotransformed into Arabidopsis protoplasts as described previously (Walter et al., 2004; Yoo et al., 2007). The protoplasts were incubated for $12-16 \mathrm{~h}$, and the fluorescence was determined using a confocal microscope (ZEISS-510Meta). The YFP fluorescence was excited by a 514-nm laser and captured at $523-600 \mathrm{~nm}$, and the chlorophyll autofluorescence was captured at $650-750 \mathrm{~nm}$.

The procedures used for pull-down assays were described previously with some modifications (Yang et al., 2008; Liu et al., 2013b). GST, GST-SIHDA1, GST-SIHDA3, and GST-SIHDA4 recombinant proteins were incubated with $30 \mathrm{ul}$ of GST resin in a binding buffer (50 mM Tris- $\mathrm{HCl}, \mathrm{pH} 7.5,100 \mathrm{mM} \mathrm{NaCl}, 0.25 \%$ Triton $\mathrm{X}-100$, and $35 \mathrm{mM} \beta$-mercaptoethanol) for $2 \mathrm{~h}$ at $4^{\circ} \mathrm{C}$, the binding reaction was washed three times with the binding buffer and then the TM29-His or TAG1-His recombinant protein was added and incubated for an additional $2 \mathrm{~h}$ at $4^{\circ} \mathrm{C}$. After extensive washing, the pulled-down proteins were eluted by boiling, separated by $10 \%$ SDS-PAGE, and detected by immunoblotting using an anti-His antibody.

\section{RESULTS}

\section{IDENTIFICATION OF HDACs IN TOMATO}

The HDACs of Arabidopsis amino acid sequences were used as queries to search against the SGN annotation database with the BLAST program. All sequences with an E-value below $10^{-2}$ were selected for further analysis. NCBI Conserved Domain Search, Pfam and SMART database were used to confirm each candidate protein sequence. Previously, 14 genes were identified as deduced HDAC genes in tomato (Cigliano et al., 2013b). We identified a new HDAC gene, SIHDA10, belonging to the RPD3/HDA1 family in the tomato genome (Table 1, Figure 1). Fifteen $H D A C$ genes distribute in different tomato chromosomes with various

Table 1 | Tomato histone deacetylase proteins.

\begin{tabular}{|c|c|c|c|c|c|c|c|}
\hline HDAC gene family & Gene name ${ }^{a}$ & Loc. symbol ${ }^{b}$ & Accession number ${ }^{c}$ & ORF length(bp) ${ }^{d}$ & protein length $^{\mathbf{e}}$ & Localization ${ }^{f}$ & Number of exons \\
\hline \multirow[t]{10}{*}{ RPD3/HDA1 } & SIHDA1 & Solyc09g091440 & XP_004247825 & 1497 & 498 & nuc & 7 \\
\hline & SIHDA3 & Solyc06g071680 & XP_004241512 & 1416 & 471 & nuc & 6 \\
\hline & SIHDA2 & Solyc03g112410 & XP_004236540 & 1350 & 449 & per & 5 \\
\hline & SIHDA4 & Solyc11g067020 & XP_004251031 & 1293 & 430 & cyto & 14 \\
\hline & SIHDA5 & Solyc08g065350 & XP_004245106 & 1044 & 347 & C & 13 \\
\hline & SIHDA9 & Solyc03g115150 & XP_004235971 & 1933 & 649 & cyto & 14 \\
\hline & SIHDA8 & Solyc03g119730 & XP_004235741 & 1833 & 610 & nuc & 17 \\
\hline & SIHDA7 & Solyc01g009110 & XP_004228472 & 798 & 265 & Plas & 4 \\
\hline & SIHDA6 & Solyc06g074080 & XP_004241339 & 1148 & 385 & cyto & 3 \\
\hline & SIHDA10 & Solyc01g009120 & XP_004228472 & 615 & 204 & C & 6 \\
\hline \multirow[t]{2}{*}{ SIR2 } & SISRT1 & Solyc07g065550 & XP_004244044 & 1419 & 472 & nuc & 14 \\
\hline & SISRT2 & Solyc04g009430 & XP_004236824 & 1158 & 385 & $\mathrm{M}$ & 11 \\
\hline \multirow[t]{3}{*}{ HD2 } & SIHDT2 & Solyc10g085560 & XP_004249622 & 924 & 307 & nuc & 11 \\
\hline & SIHDT3 & Solyc11g066840 & XP_004251012 & 954 & 317 & nuc & 7 \\
\hline & SIHDT1 & Solyc09g009030 & XP_004246566 & 810 & 269 & nuc & 10 \\
\hline
\end{tabular}

a Systematic designation given to tomato histone deacetylase.

${ }^{b}$ Accession number of SGN (http://solgenomics.net) locus ID.

${ }^{c}$ Accession numbers of full-length protein sequence available at NCBI (http://www.ncbi.nlm.nih.gov/).

${ }^{d}$ Length of open reading frame (number of base pair).

e Length of protein (number of amino acid).

${ }^{f}$ Localization of tomato histone deacetylase proteins supported by WoLF PSORT (http://www.genscript.com/psort/wolf_psort.html).

Nuc, nucleus; per, peroxisome; Plas, plasma membrane; cyto, cytoplasm; $M$, mitochondrion; $C$, chloroplast. 
numbers of exons (Table 1). To further investigate the evolutionary relationships, phylogenetic analyses were performed using the Mega 3.0 software. The phylogenetic tree indicates that 15 HDACs in tomato can be divided into three clades: RPD3/HDA1, SIR2, and HD2 families (Table 1, Figure 1A). There are 10 members in the RPD3/HDA1 family, which can be further divided into four subclasses as Class I, Class II, Class III, and Class IV. In addition, there are two and three members in the SIR2 and HD2 family, respectively.

The conserved domains were predicted with hammerbased website Smart and Pfam (Figure 1B). The members of RPD3/HDA1 family all have a deacetylase catalytic domain. Both SIHDA 1 and SIHDA8 have the zinc-finger domain which might be involved in the binding of DNA. The N-terminus of the HD2 family contains the conserved typical pentapeptide motif (MEFWG) (see Supplementary Figure 1). In addition, SIHDT2 and SIHDT1 both contain a $\mathrm{C}_{2} \mathrm{H}_{2}$ zinc-finger that might be involved in protein-protein interaction. Furthermore, the members of SIR2 family have the conserved domain that is dependent on NAD.

\section{THE EXPRESSION PATTERN OF SLHDACS}

Considering the typical fruit development and maturation processes of tomato, we focused on the several important stages

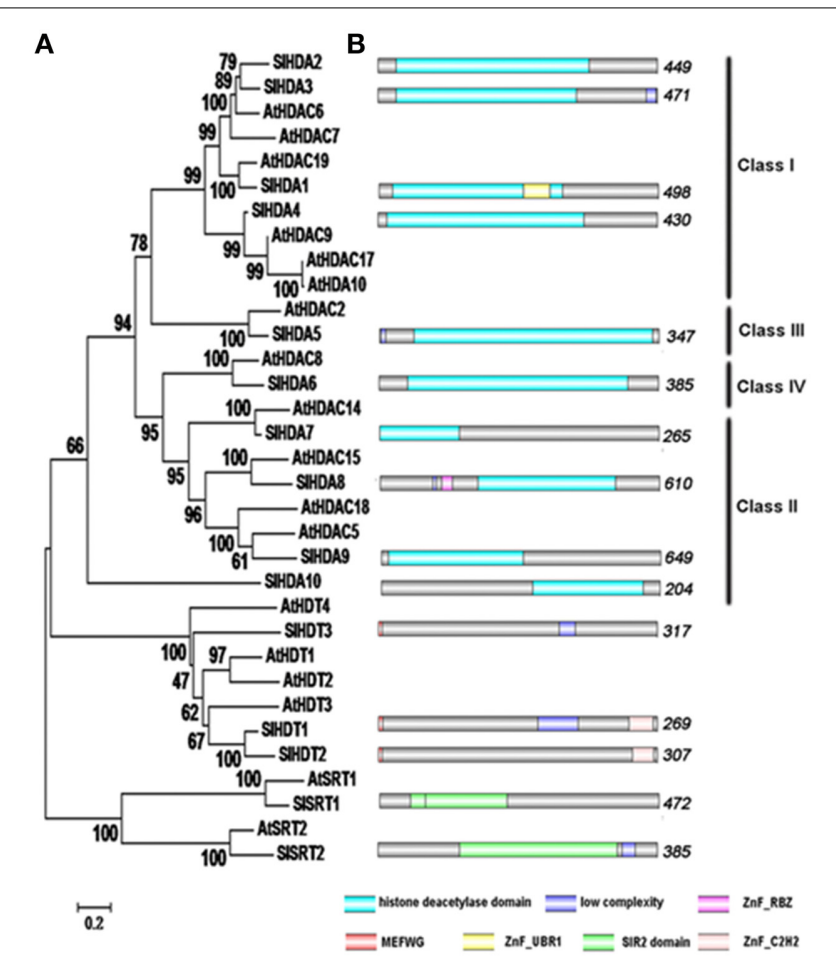

FIGURE 1 | Phylogenetic analysis and domain organization of HDACs in tomato. (A) The neighbor joining phylogenetic tree constructed by MEGA 3 refers to the evolutionary relationship between the HDACs in tomato and Arabidopsis. (B) Domain architecture of the HDACs in tomato was drawn by DOG 2.0. The location and size of domains are shown by different color as indicated. The proteins belonging to each family are grouped together. such as fruits on 10,20, and 30 days post anthesis (dpa) as well as fruits of the maturing green stage (MG), breaker stage, turning stage, pink stage and red ripe stage (Teyssier et al., 2008) (see Supplementary Figure 2). Except for SlHDA2, the transcripts of other 14 SlHDACs were detectable in roots, hypocotyls, cotyledons, euphylla, leaves and fruits from $10 \mathrm{dpa}$ to the red ripe stage (Figure 2). The Class I members of RPD3/HDA1 family, SlHDA3, SlHDA1, and SlHDA4, were all highly expressed in flowers but lowly expressed in later fruit stages. In addition, SlHDA1 was also highly expressed in the red ripe stage (Figure 2). The transcript of the Class III member, SlHDA5, accumulated to a high level in flowers and $10 \mathrm{dpa}$, but decreased as fruit development and ripening (Figure 2). The SlHDA6, a member of Class IV, was highly expressed in the red ripe stage but lower expressed in cotyledons, flowers and fruits at 10, 20, and $30 \mathrm{dpa}$ stages. Two members of Class II subfamily, SlHDA7 and SIHDA10, shared the similar expression pattern and were highly expressed in cotyledons, euphylla and leaves (Figure 2). Unlike SIHDA7 and SIHDA10, the other members of Class II subfamily, SIHDA 8 and SIHDA9, showed different expression patterns. SlHDA 8 was highly expressed in $10 \mathrm{dpa}$ stage, while the SIHDA9 transcript was accumulated in cotyledons and euphylla (Figure 2).

In comparison, the two members of HD2 family, SlHDT3 and SlHDT1, were highly expressed in roots and hypocotyls. In addition, transcripts of SlHDT2 and SlHDT1 were also accumulated in flowers and $10 \mathrm{dpa}$ stage (Figure 2). Furthermore, the members of SIR2 family, SISRT1 and SISRT2, were highly expressed in cotyledons, flowers and $10 \mathrm{dpa}$ stage (Figure 2).

\section{THE SUBCELLULAR LOCALIZATION OF SLHDACs}

WoLF PSORT was used to determine the predicted subcellular localizations of SIHDACs. Interestingly, SIHDACs were predicted to have various subcellular localizations including nuclei, peroxisomes, plasma membranes, cytoplasms, mitochondria and chloroplasts (Table 1).

To further determine the subcellular localization of SIHDACs, the cDNA of each SIHDAC was fused with Yellow Fluorescent Protein (YFP) driven by the Cauliflower mosaic virus 35S promoter and transiently expressed in Arabidopsis protoplasts. As shown in Figure 3, SIHDACs of the three subfamilies showed different subcellular localization. The members of the RPD3/HDA1 subfamily displayed variable subcellular localizations. SIHDA1, SIHDA5, and SIHDA8 were localized in the nucleus, while SIHDA9 was only localized in the cytoplasm (Figure 3A). In contrast, SIHDA3 and SIHDA4 were localized in both the cytosol and nucleus, implying the possibility of shuttling between the nucleus and cytoplasm. Interestingly, SIHDA10 was localized in the chloroplast, which is consistent with the result of the WoLF PSORT programs analysis.

Consistent with the predicted localization using WoLF PSORT programs, SISRT1 and all the three members of HD2 subfamily were localized in the nucleus (Table 1, Figures 3B,C). A recent study showed that the SRT2 in Arabidopsis was localized at the inner mitochondrial membrane (Konig et al., 2014). Nevertheless, SISRT2, the homolog of Arabidopsis SRT2 in tomato, was localized in both the nucleus and cytosol (Figure 3C). 

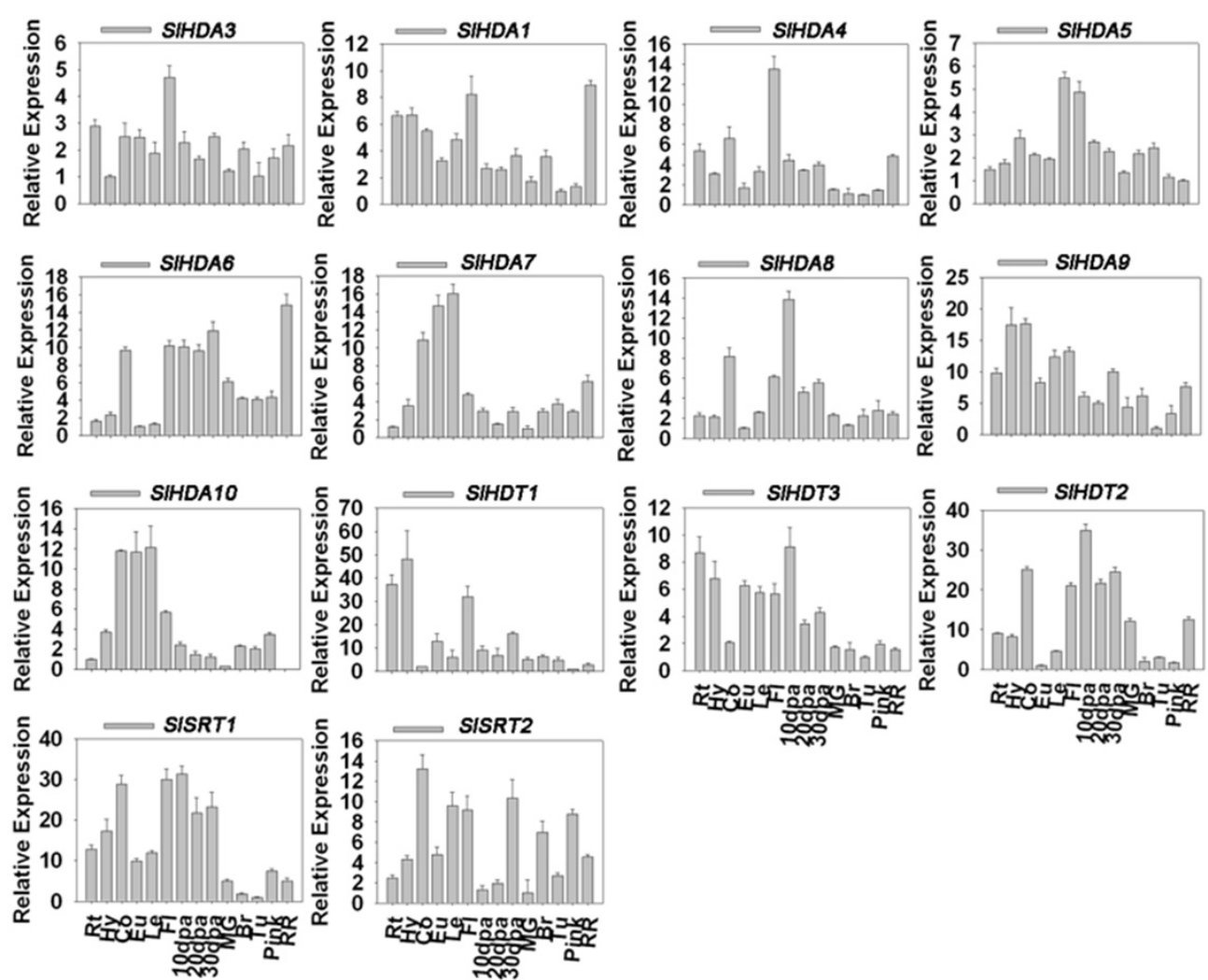

FIGURE 2 | Quantitative RT-PCR analysis of the expression of HDACs in different tissues and developmental stages. Roots (Rt), hypocotyls (Hy), cotyledons (Co), euphylla (Eu), and leaves (Le) of 5-week-old plate cultured plants and flowers (FI), fruits at 10 days post anthesis (10 dpa), $20 \mathrm{dpa}, 30 \mathrm{dpa}$,

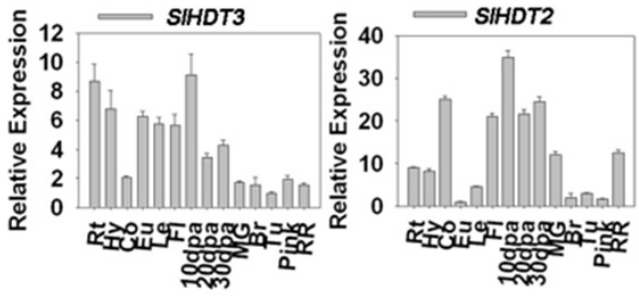

mature green stage (MG), break stage ( $\mathrm{Br})$, turning stage (Tu), pink stage (pink), and red ripe stage (RR) were collected for total RNA isolation. RT-PCR was amplified using gene-specific primers. The tomato Actin

(Solyc03g078400) was used as an internal control.
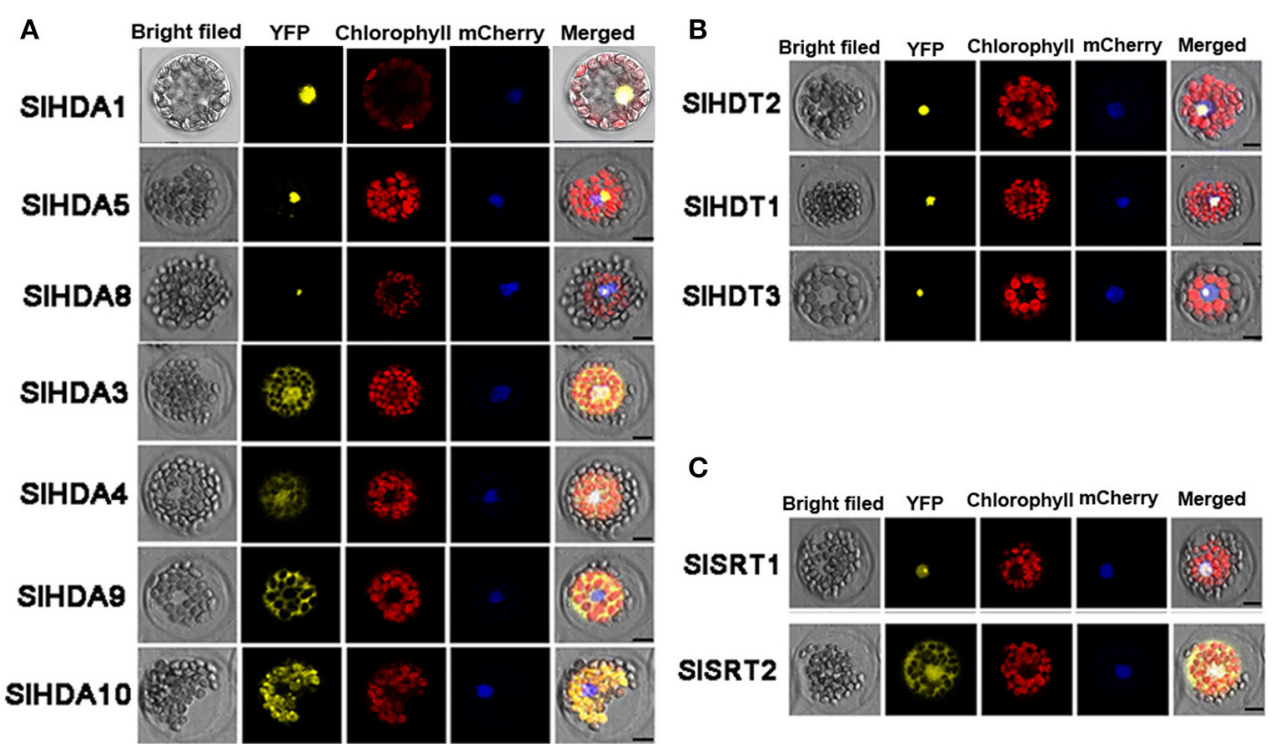

FIGURE 3 | Protoplast transient expression analysis using HDAC-YFP fusion constructs. Subcellular location of RPD3/HDA1 (A), HD2 (B), and SRT2 (C) family HDACs was determined via Arabidopsis protoplast PEG transfection using HDAC-YFP fusion constructs. Red color indicated autofluorescence emitted by chloroplasts. The blue color indicates the nucleus using the mCherry as the nuclear marker. Bar represents $7.5 \mu \mathrm{m}$. 


\section{MEMBERS OF RPD3/HDA1 SUBFAMILY INTERACT WITH MADS-BOX PROTEINS}

In Arabidopsis, two MADS-box proteins associated with flowering, AGAMOUS-like 15 (AGL15) and AGAMOUS-like 24 (AGL24), were shown to interact with SAP18, a subunit of the SIN3-HDAC complex involved in transcriptional repression (Hill et al., 2008; Liu et al., 2009). Furthermore, a recent study found that SIMADS1/LeMADS1 interacted with the Nterminal domain of mammalian HDAC5 in vitro (Gaffe et al., 2011). These data suggest that MADS transcription factors may be associated with a HDAC protein complex. The interactions between the members of RPD3/HDA1 family HDACs and the MADS-box proteins involved in fruit development were performed using yeast two-hybrid assays. Our results show that two MADS-box proteins, TM29 and TAG1, interacted with SIHDA1 and SIHDA4 (Figure 4A). In addition, SIHDA3, the homolog of Arabidopsis HDA6 in tomato, also interacted with TAG1 in yeast cells (Figure 4A). Interestingly, we also observed TM29 and TAG1 interacted with SIHDA6 and SIHDA7 in yeast cells (see Supplementary Figure 3).

The interaction of SIHDACs and these MADS-box proteins was further studied in vivo by the bimolecular fluorescence complementation (BiFC) and in vitro pull-down assays. For BiFC assays, SIHDACs and the MADS-box proteins were fused to either the N-terminal or C-terminal portion of YFP in the pSAT1 vector. The constructs were codelivered into Arabidopsis protoplasts and then incubated under light for $12 \mathrm{~h}$. Strong YFP signal was observed in the nucleus when transiently coexpressing SlHDA1-YN/ SlHDA3-YN/ SlHDA4-YN/ with TAG1-YC (Figure 4B). Similarly, strong YFP signal was also observed in the nucleus when transiently coexpressing SIHDA1YN/ SlHDA4-YN/ with TM29-YC (Figure 4B). As a control, no YFP signal was observed when these construct were cotransformed with the YN or NC empty vector (see Supplementary Figure 4).

For in vitro pull-down assays, purified TM29-His or TAG1His recombinant protein was incubated with Glutathione S-Transferase (GST)-SIHDA1, GST-SIHDA3 or GST-SIHDA4 protein, respectively. As shown in Figure 4C, TM29-His was pulled down by GST-SIHDA1 and GST-SIHDA4. Similarly, TAG1-His was also pulled down by GST-SIHDA1, GST-SIHDA4 and GST-SIHDA3. These results indicate that TM29 is directly associated with SIHDA1 and SIHDA4, whereas TAG1 is directly associated with SIHDA1, SIHDA3 and SIHDA4.

\section{DISCUSSION}

In Arabidopsis, HDACs were found to be crucial players in all aspects of plant development including embryogenesis, abaxial/adaxial polarity determination, flowering, senescence, responses to day length and environmental stresses (Hollender and Liu, 2008; Ma et al., 2013; Liu et al., 2014). In comparison, relatively few HDACs were characterized in other plant species. In this study, $15 \mathrm{HDACs}$ in tomato were identified using the bioinformatics analysis. The SIHDACs can be separated into three families: RPD3/HDA1, HD2 and SIR2 (Figure 1). Ten SIHDACs belong to the RPD3/HDA1 family and they all contain a typical

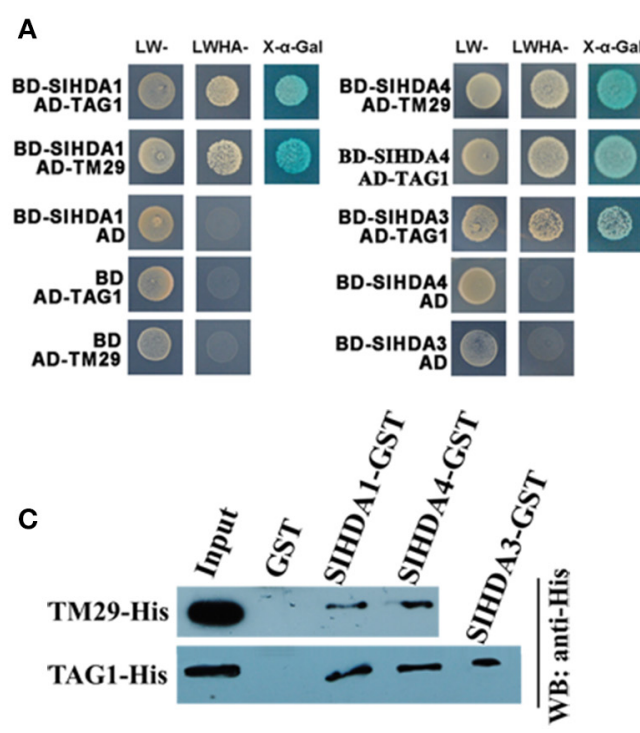

FIGURE 4 | SIHDACs interacted with MDAS-box proteins, TM29 and TAG1. (A) SIHDACs interacted with TM29 and TAG1 in yeast two-hybrid assays. SIHDA1, SIHDA3, and SIHDA4 were cloned into pGADT7 vector, whereas TM29 and TAG1 were cloned into pGBKT7 vector, respectively. The plasmids were cotransformed into the yeast strain $\mathrm{AH} 109$. The transformants were grown on the selective minimal medium without Leu and Trp (LW-) or without Leu, Trp, Ade and His (LWHA-). SIHDACs interacted with TM29 and TAG1 in Arabidopsis protoplasts in BiFC assays. (B) SIHDA1/SIHDA2/SIHDA4 fused with the $\mathrm{N}$ terminus (YN) and TAG1/TM29 fused with the $\mathrm{C}$ terminus

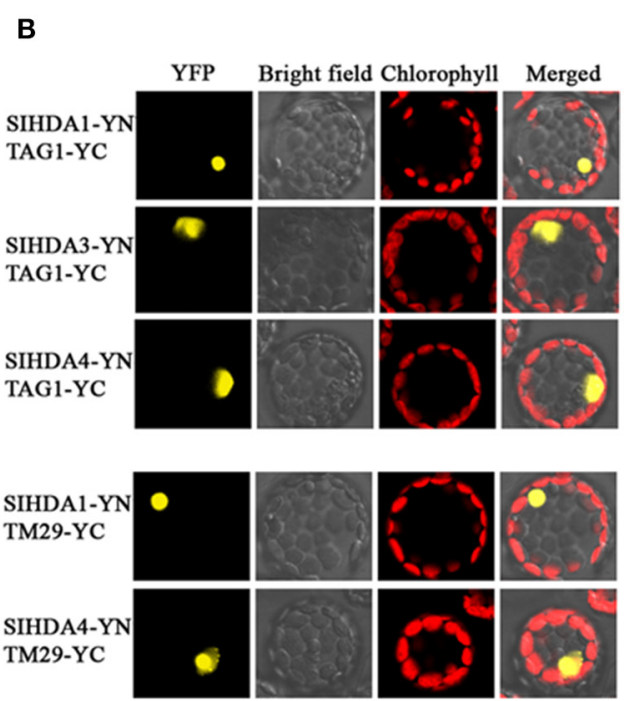

(YC) of YFP were cotransformed into protoplasts and then incubated in the $100 \mu \mathrm{mol} . \mathrm{m}-2 . \mathrm{s}-1$ for $12 \mathrm{~h}$. The fluorescence was determined using a confocal microscope. The YFP fluorescence was excited by a $514 \mathrm{~nm}$ laser and captured at 523-600 $\mathrm{nm}$, and the chlorophyll autofluorescence was captured at 650-750 $\mathrm{nm}$. Bar $=20 \mu \mathrm{m}$. SIHDACs interacted with TM29 and TAG1 in pull-down assays. (C) GST- SIHDAC1, GST- SIHDAC3, GST- SIHDAC4 or GST was incubated with either TM29-His or TAG1-His and GST affinity resin, and the bound proteins were then eluted from resin and probed with the anti-His antibody. 
deacetylase catalytic domain that is essential for the deacetylation activity (Figure 1B). In addition, the two members of the SIR2 family do not share sequence homology with other HDAC family members (Figure 1B). The three members of the HD2 family in tomato all contain a conserved pentapeptide (MEFWG) on the $\mathrm{N}$-terminus of the protein, while the $\mathrm{C}$-terminus has a variant domain (Additional file 1). $\mathrm{A}_{2} \mathrm{H}_{2}$ zinc finger domain was identified on the C-terminus of the SIHDT1 and SIHDT2, which may participate in the protein-protein interaction (Figure 1). Compared with the previous report (Cigliano et al., 2013b), a new HDAC protein, SIHDA10, belonging to the RPD3/HDA1 family, was also found in the tomato (Table 1, Figure 1). The Conserved Domain search result clearly showed that SIHDA10 contains an Arginase HDAC domain (Accession NO. cl17011).

The development progress of tomato can be divided into several stages after the flower anthesis. Mature green (MG) stage, breaker stage $(\mathrm{Br})$, turning stage $(\mathrm{Tu})$ and the final red ripe stage (RR) are all the key development stages of tomato. MG stages can also be divided into different stages according to the gel production in locules, seed maturation and the external color (Teyssier et al., 2008). The fruit ripening is regulated by numerous genes which participate in different pathways influencing texture, color, pigment and aroma. Previous RNA-seq expression data demonstrate that SlHDACs may play roles in tomato fruit ripening, since SIHDA1 and SIHDA3 are highly expressed at B10 (10 days after breaking) and B fruit stages, respectively (Sato et al., 2012; Cigliano et al., 2013b). Consistent with this report, our data show that SIHDA4, SIHDA8, SIHDA6, and SlHDT2 were all highly expressed in inflorescences to fruits development stages, indicating the potential role of SlHDACs in fruits development (Figure 2). SlHDA3, the close homolog of AtHDA6 (Tanaka et al., 2008; Chen et al., 2010; Yu et al., 2011), was constitutively expressed in all stage of tomato development, especially in flowers (Figure 2). The two members of the SIR2 family show markedly different expression profiles, implying that they may be involved in different cellular processes (Figure 2). We also found that the expression profiles of some SlHDACs were not consistent with previous data based on RNA-seq analysis (Cigliano et al., 2013b). The discrepancy may be derived from the fact that plant materials at different developmental stages were used for the gene expression analysis. Interestingly, we found that the transcript of SIHDA2 was not detected in all tissues and fruits of different development stages analyzed. Similarly, previous RNA-seq data also showed that the transcript level of SIHDA2 is very low in tomato (Sato et al., 2012).

We found that the members of tomato RPD3/HDA1 family show a variety of subcellular localizations (Figure 3). SIHDA1, SIHDA5, and SIHDA8 were localized in the nucleus. In contrast, SIHDA3 and SIHDA4 were localized in both the nucleus and cytoplasm, suggesting the possibility of shuttling between the nucleus and cytoplasm. The shuttling of HDACs between the nucleus and the cytoplasm was previously reported in Arabidopsis and mammalian cells (Sengupta and Seto, 2004; Alinsug et al., 2009). In contrast, SIHDA9, the member Class II HDACs, was only localized in cytoplasm, implying that its substrates may be cytoplasmic proteins. Interestingly, SIHDA10 was localized in the chloroplast and the SIHDA10 transcript was highly expressed in leaf tissues. It remains to be determined whether SIHDA10 deacetylates chloroplast proteins. Like their Arabidopsis homologs, the members of tomato HD2 subfamily were all localized in the nucleus (Zhou et al., 2004). Previous studies indicate that the Arabidopsis AtSRT2 is localized mitochondria (Konig et al., 2014). In contrast, we found that SISRT2 was localized in both the nucleus and cytoplasm. The difference in subcellular localization suggests that different members of the same HDAC family may have distinctive functions in tomato.

To date, a number of MADS-box proteins attributing to fruit ripening of tomato have been identified (Vrebalov et al., 2002, 2009; Victoria et al., 2003; Giovannoni, 2007; Dong et al., 2013). In Arabidopsis, it was reported that the MADS-box proteins, AGL15 and AGL24, can interact with SAP18, a component of the SIN3/HDAC complex involved in transcriptional repression (Hill et al., 2008; Liu et al., 2009), suggesting that these MADS transcription factors may be associated with a HDAC protein complex. A previous study indicates that the tomato MADS protein SIMADS1/LeMADS1 interacts with the N-terminal domain of the mammalian HDAC5 in vitro (Gaffe et al., 2011). Our data show that SIHDA1 and SIHDA4 interacted TAG1 and TM29, the MADS-box proteins associated with tomato reproductive development (Figure 4). TAG1 is a member of the AGAMOUS clade of MADS-box genes in tomato and it is expressed in flowers and ripening fruits. Suppression of the TAG1 gene in tomato leads to a variety of floral defects and production of smaller amounts of pollen (Pan et al., 2010). In addition, TAG1 is necessary for the expression of both ethylene-dependent and independent genes during ripening (Klee and Giovannoni, 2011). On the other hand, TM29, a tomato SEPALLATA homolog, is highly expressed in the primordia of all four whorls of floral organs (AmpomahDwamena et al., 2002). Down-regulation of TM29 via cosuppression or antisense techniques causes parthenocarpic fruits and aberrant flowers, as the petals and stamens are partially converted to a sepaloid identity (Ampomah-Dwamena et al., 2002). SIHDAC1, SIHDAC4, TAG1, and TM29 all have peak expression at the flowering stage and all of them are expressed during fruit development (Ampomah-Dwamena et al., 2002; Pan et al., 2010) (See Supplementary Figure 5). The interaction of TAG1 and TM29 with SIHDA1 and SIHDA4 indicates that these MADAbox proteins may recruit HDACs to regulate gene expression in reproductive development in tomato.

\section{CONCLUSION}

Fifteen SIHDACs identified from the genome of Solanum lycopersicum can be divided into RPD3/HDA1, SIR2, and HD2 families. Most SlHDACs were expressed in all tissues examined with different transcript abundance. SIHDA8, SIHDA1, SIHDA5, SISRT1 and members of the HD2 family were localized to the nucleus, whereas SIHDA3 and SIHDA4 were localized in both the cytoplasm and nucleus. Furthermore, TAG1 and TM29 interacted with SIHDA1 and SIHDA4, indicating that these MADA-box proteins may recruit HDACs to regulate gene expression in reproductive development in tomato.

\section{AUTHOR CONTRIBUTIONS}

Songguang Yang and Keqiang Wu conceived this project and designed all research. Linmao Zhao, Jingxia Lu, Pei-Ying Wu, and Jianxia Zhang performed the research. Songguang Yang and 
Keqiang $\mathrm{Wu}$ and Jingxia $\mathrm{Lu}$ and Linmao Zhao analyzed data. Songguang Yang and Keqiang Wu wrote the article.

\section{ACKNOWLEDGMENTS}

We are grateful to Dr. Ying Wang (South China Botanical Garden) for providing the seeds of Solanum lycopersicum cultivar Henz1706. We thank Technology Commons, College of Life Science, National Taiwan University for the convenient use of the Bio-Rad real-time PCR system and the confocal spectral microscope imaging system. This work was supported by grants from the National Basic Research Program of China (973 program No. 2012CB910900), National Natural Science Foundation of China (No. 31201106, No. 31128001 and No. 31371308). This work was also supported by the Ministry of Science and Technology of Taiwan (101-2923-B-002-005-MY3, 101-2311-B002-012-MY3 and 102-2321-B-002-039-) and National Taiwan University (103R892005).

\section{SUPPLEMENTARY MATERIAL}

The Supplementary Material for this article can be found online at: http://www.frontiersin.org/journal/10.3389/fpls.2014.00760/ abstract

\section{REFERENCES}

Alinsug, M. V., Yu, C. W., and Wu, K. Q. (2009). Phylogenetic analysis, subcellular localization, and expression patterns of RPD3/HDA1 family histone deacetylases in plants. BMC Plant Biol. 9:37. doi: 10.1186/1471-2229-9-37

Allfrey, V. G., Faulkner, R., and Mirsky, A. E. (1964). Acetylation and methylation of histones and their possible role in regulation of Rna synthesis. Proc. Natl. Acad. Sci. U.S.A. 51, 786-794. doi: 10.1073/pnas.51.5.786

Ampomah-Dwamena, C., Morris, B. A., Sutherland, P., Veit, B., and Yao, J. L. (2002). Down-regulation of TM29, a tomato SEPALLATA homolog, causes parthenocarpic fruit development and floral reversion. Plant Physiol. 130, 605-617. doi: 10.1104/pp.005223

Aufsatz, W., Mette, M. F., Van Der Winden, J., Matzke, M., and Matzke, A. J. M. (2002). HDA6, a putative histone deacetylase needed to enhance DNA methylation induced by double-stranded RNA. EMBO J. 21, 6832-6841. doi: 10.1093/emboj/cdf663

Bourque, S., Dutartre, A., Hammoudi, V., Blanc, S., Dahan, J., Jeandroz, S., et al. (2011). Type-2 histone deacetylases as new regulators of elicitorinduced cell death in plants. New Phytol. 192, 127-139. doi: 10.1111/j.14698137.2011.03788.x

Brownell, J. E., Zhou, J. X., Ranalli, T., Kobayashi, R., Edmondson, D. G., Roth, S. Y., et al. (1996). Tetrahymena histone acetyltransferase A: a homolog to yeast Gcn5p linking histone acetylation to gene activation. Cell 84, 843-851. doi: 10.1016/S0092-8674(00)81063-6

Busconi, M., Reggi, S., Fogher, C., and Bavaresco, L. (2009). Evidence of a sirtuin gene family in grapevine (Vitis vinifera L.). Plant Physiol. Biochem. 47, 650-652. doi: 10.1016/j.plaphy.2009.03.003

Campos, E. I., and Reinberg, D. (2009). Histones: annotating chromatin. Annu. Rev. Genet. 43, 559-599. doi: 10.1146/annurev.genet.032608.103928

Chen, L. T., Luo, M., Wang, Y. Y., and Wu, K. Q. (2010). Involvement of Arabidopsis histone deacetylase HDA6 in ABA and salt stress response. J. Exp. Bot. 61, 3345-3353. doi: 10.1093/jxb/erq154

Cigliano, R. A., Cremona, G., Paparo, R., Termolino, P., Perrella, G., Gutzat, R., et al. (2013a). Histone deacetylase AtHDA7 is required for female gametophyte and embryo development in Arabidopsis. Plant Physiol. 163, 431-440. doi: 10.1104/pp.113.221713

Cigliano, R. A., Sanseverino, W., Cremona, G., Ercolano, M. R., Conicella, C., and Consiglio, F. M. (2013b). Genome-wide analysis of histone modifiers in tomato: gaining an insight into their developmental roles. MBC Genomics 14:57. doi: 10.1186/1471-2164-14-57

Demetriou, K., Kapazoglou, A., Tondelli, A., Francia, E., Stanca, M. A., Bladenopoulos, K., et al. (2009). Epigenetic chromatin modifiers in barley: I. Cloning, mapping and expression analysis of the plant specific HD2 family of histone deacetylases from barley, during seed development and after hormonal treatment. Physiol. Plant. 136, 358-368. doi: 10.1111/j.1399-3054.2009.01236.x

Dong, T. T., Hu, Z. L., Deng, L., Wang, Y., Zhu, M. K., Zhang, J. L., et al. (2013). A tomato MADS-box transcription factor, SIMADS1, acts as a negative regulator of fruit ripening. Plant Physiol. 163, 1026-1036. doi: 10.1104/pp.113.224436

Ferrandiz, C., Liljegren, S. J., and Yanofsky, M. F. (2000). Negative regulation of the SHATTERPROOF genes by FRUITFULL during Arabidopsis fruit development. Science 289, 436-438. doi: 10.1126/science.289.5478.436

Gaffe, J., Lemercier, C., Alcaraz, J. P., and Kuntz, M. (2011). Identification of three tomato flower and fruit MADS-box proteins with a putative histone deacetylase binding domain. Gene 471, 19-26. doi: 10.1016/j.gene.2010.10.002

Giovannoni, J. J. (2007). Fruit ripening mutants yield insights into ripening control. Curr. Opin. Plant Biol. 10, 283-289. doi: 10.1016/j.pbi.2007.04.008

Girin, T., Paicu, T., Stephenson, P., Fuentes, S., Korner, E., O’brien, M., et al. (2011). INDEHISCENT and SPATULA interact to specify carpel and valve margin tissue and thus promote seed dispersal in Arabidopsis. Plant Cell 23, 3641-3653. doi: 10.1105/tpc.111.090944

Groszmann, M., Paicu, T., Alvarez, J. P., Swain, S. M., and Smyth, D. R. (2011). SPATULA and ALCATRAZ, are partially redundant, functionally diverging bHLH genes required for Arabidopsis gynoecium and fruit development. Plant J. 68, 816-829. doi: 10.1111/j.1365-313X.2011.04732.x

Gu, Q., Ferrandiz, C., Yanofsky, M. F., and Martienssen, R. (1998). The FRUITFULL MADS-box gene mediates cell differentiation during Arabidopsis fruit development. Development 125, 1509-1517.

Haigis, M. C., and Guarente, L. P. (2006). Mammalian sirtuins-emerging roles in physiology, aging, and calorie restriction. Genes Dev. 20, 2913-2921. doi: 10.1101/gad.1467506

Hill, K., Wang, H., and Perry, S. E. (2008). A transcriptional repression motif in the MADS factor AGL15 is involved in recruitment of histone deacetylase complex components. Plant J. 53, 172-185. doi: 10.1111/j.1365-313X.2007.03336.x

Hollender, C., and Liu, Z. C. (2008). Histone deacetylase genes in Arabidopsis development. J. Integr. Plant Biol. 50, 875-885. doi: 10.1111/j.17447909.2008.00704.x

Klee, H. J., and Giovannoni, J. J. (2011). Genetics and control of tomato fruit ripening and quality attributes. Ann. Rev. Genet. 45, 41-59. doi: 10.1146/annurevgenet-110410-132507

Konig, A. C., Hartl, M., Pham, P. A., Laxa, M., Boersema, P. J., Orwat, A., et al. (2014). The Arabidopsis class II sirtuin is a lysine deacetylase and interacts with mitochondrial energy metabolism. Plant Physiol. 164, 1401-1414. doi: 10.1104/pp.113.232496

Kumar, S., Tamura, K., and Nei, M. (2004). MEGA3: integrated software for molecular evolutionary genetics analysis and sequence alignment. Brief. Bioinform. 5 , 150-163. doi: 10.1093/bib/5.2.150

Lagace, M., Chantha, S. C., Major, G., and Matton, D. P. (2003). Fertilization induces strong accumulation of a histone deacetylase (HD2) and of other chromatin-remodeling proteins in restricted areas of the ovules. Plant Mol. Biol. 53, 759-769. doi: 10.1023/B:PLAN.0000023665.36676.89

Liu, C., Li, L. C., Chen, W. Q., Chen, X., Xu, Z. H., and Bai, S. N. (2013a). HDA18 Affects cell fate in Arabidopsis root epidermis via histone acetylation at four kinase genes. Plant Cell 25, 257-269. doi: 10.1105/tpc.112.107045

Liu, C., Xi, W. Y., Shen, L. S., Tan, C. P., and Yu, H. (2009). Regulation of floral patterning by flowering time genes. Dev. Cell 16, 711-722. doi: 10.1016/j.devcel.2009.03.011

Liu, X. C., Chen, C. Y., Wang, K. C., Luo, M., Tai, R., Yuan, L. Y., et al. (2013b). PHYTOCHROME INTERACTING FACTOR3 associates with the histone deacetylase HDA15 in repression of chlorophyll biosynthesis and photosynthesis in etiolated Arabidopsis seedlings. Plant Cell 25, 1258-1273. doi: 10.1105/tpc.113.109710

Liu, X. C., Yang, S. G., Zhao, M. L., Luo, M., Yu, C. W., Chen, C. Y., et al. (2014). Transcriptional repression by histone deacetylases in plants. Mol. Plant 7, 764-772. doi: 10.1093/mp/ssu033

Long, J. A., Ohno, C., Smith, Z. R., and Meyerowitz, E. M. (2006). TOPLESS regulates apical embryonic fate in Arabidopsis. Science 312, 1520-1523. doi: 10.1126/science. 1123841

Luger, K., Mader, A. W., Richmond, R. K., Sargent, D. F., and Richmond, T. J. (1997). Crystal structure of the nucleosome core particle at 2.8 angstrom resolution. Nature 389, 251-260. doi: 10.1038/38444

Lusser, A., Kolle, D., and Loidl, P. (2001). Histone acetylation: lessons from the plant kingdom. Trends Plant Sci. 6, 59-65. doi: 10.1016/S1360-1385(00)01839-2 
Ma, X. J., Lv, S. B., Zhang, C., and Yang, C. P. (2013). Histone deacetylases and their functions in plants. Plant Cell Rep. 32, 465-478. doi: 10.1007/s00299-01 3-1393-6

Nagy, L., Kao, H. Y., Chakravarti, D., Lin, R. J., Hassig, C. A., Ayer, D. E., et al. (1997). Nuclear receptor repression mediated by a complex containing SMRT, mSin3A, and histone deacetylase. Cell 89, 373-380. doi: 10.1016/S00928674(00)80218-4

Pan, I. L., Mcquinn, R., Giovannoni, J. J., and Irish, V. F. (2010). Functional diversification of AGAMOUS lineage genes in regulating tomato flower and fruit development. J. Exp. Bot. 61, 1795-1806. doi: 10.1093/jxb/erq046

Pandey, R., Muller, A., Napoli, C. A., Selinger, D. A., Pikaard, C. S., Richards, E. J., et al. (2002). Analysis of histone acetyltransferase and histone deacetylase families of Arabidopsis thaliana suggests functional diversification of chromatin modification among multicellular eukaryotes. Nucleic Acids Res. 30, 5036-5055. doi: $10.1093 /$ nar/gkf660

Probst, A. V., Fagard, M., Proux, F., Mourrain, P., Boutet, S., Earley, K., et al. (2004). Arabidopsis histone deacetylase HDA6 is required for maintenance of transcriptional gene silencing and determines nuclear organization of rDNA repeats. Plant Cell 16, 1021-1034. doi: 10.1105/tpc.018754

Ren, J., Wen, L. P., Gao, X. J., Jin, C. J., Xue, Y., and Yao, X. B. (2009). DOG 1.0: illustrator of protein domain structures. Cell Res. 19, 271-273. doi: $10.1038 / \mathrm{cr} .2009 .6$

Sato, S., Tabata, S., Hirakawa, H., Asamizu, E., Shirasawa, K., Isobe, S., et al. (2012). The tomato genome sequence provides insights into fleshy fruit evolution. Nature 485, 635-641. doi: 10.1038/nature11119

Sengupta, N., and Seto, E. (2004). Regulation of histone deacetylase activities. J. Cell. Biochem. 93, 57-67. doi: 10.1002/jcb.20179

Tanaka, M., Kikuchi, A., and Kamada, H. (2008). The Arabidopsis histone deacetylases HDA6 and HDA19 contribute to the repression of embryonic properties after germination. Plant Physiol. 146, 149-161. doi: 10.1104/pp.107.111674

Teyssier, E., Bernacchia, G., Maury, S., Kit, A. H., Stammitti-Bert, L., Rolin, D., et al. (2008). Tissue dependent variations of DNA methylation and endoreduplication levels during tomato fruit development and ripening. Planta 228, 391-399. doi: 10.1007/s00425-008-0743-z

Tian, L., and Chen, Z. J. (2001). Blocking histone deacetylation in Arabidopsis induces pleiotropic effects on plant gene regulation and development. Proc. Natl. Acad. Sci. U.S.A. 98, 7647-7647. doi: 10.1073/pnas.98.1.200

Tian, L., Wang, J. L., Fong, M. P., Chen, M., Cao, H. B., Gelvin, S. B., et al. (2003). Genetic control of developmental changes induced by disruption of Arabidopsis histone deacetylase 1 (AtHD1) expression. Genetics 165, 399-409.

Tran, H. T., Nimick, M., Uhrig, R. G., Templeton, G., Morrice, N., Gourlay, R., et al. (2012). Arabidopsis thaliana histone deacetylase 14 (HDA14) is an alphatubulin deacetylase that associates with PP2A and enriches in the microtubule fraction with the putative histone acetyltransferase ELP3. Plant J. 71, 263-272. doi: 10.1111/j.1365-313X.2012.04984.x

Tzfira, T., Tian, G. W., Lacroix, B., Vyas, S., Li, J. X., Leitner-Dagan, Y., et al. (2005). pSAT vectors: a modular series of plasmids for autofluorescent protein tagging and expression of multiple genes in plants. Plant Mol. Biol. 57, 503-516. doi: 10.1007/s11103-005-0340-5

Ueno, Y., Ishikawa, T., Watanabe, K., Terakura, S., Iwakawa, H., Okada, K., et al. (2007). Histone deacetylases and ASYMMETRIC LEAVES2 are involved in the establishment of polarity in leaves of Arabidopsis. Plant Cell 19, 445-457. doi: 10.1105/tpc.106.042325

Untergasser, A., Cutcutache, I., Koressaar, T., Ye, J., Faircloth, B. C., Remm, M., et al. (2012). Primer3-new capabilities and interfaces. Nucleic Acids Res. 40:e115. doi: $10.1093 / \mathrm{nar} / \mathrm{gks} 596$

Victoria, B. M., Claudia, B., Cecilia, D., Mauricio, H. C., Silvana, B. B., Estela, V. M., et al. (2003). MADS-box genes expressed during tomato seed and fruit development. Plant Mol. Biol. 52, 801-815. doi: 10.1023/A:1025001402838
Vrebalov, J., Pan, I. L., Arroyo, A. J. M., Mcquinn, R., Chung, M., Poole, M., et al. (2009). Fleshy fruit expansion and ripening are regulated by the tomato SHATTERPROOF gene TAGL1. Plant Cell 21, 3041-3062. doi: 10.1105/tpc.109.066936

Vrebalov, J., Ruezinsky, D., Padmanabhan, V., White, R., Medrano, D., Drake, R., et al. (2002). A MADS-box gene necessary for fruit ripening at the tomato ripening-inhibitor (Rin) locus. Science 296, 343-346. doi: 10.1126/science. 1068181

Walter, M., Chaban, C., Schutze, K., Batistic, O., Weckermann, K., Nake, C., et al. (2004). Visualization of protein interactions in living plant cells using bimolecular fluorescence complementation. Plant J. 40, 428-438. doi: 10.1111/j.1365313X.2004.02219.x

Wu, K. Q., Tian, L. N., Malik, K., Brown, D., and Miki, B. (2000). Functional analysis of HD2 histone deacetylase homologs in Arabidopsis thaliana. Plant J. 22, 19-27. doi: 10.1046/j.1365-313x.2000.00711.x

Xu, C. R., Liu, C., Wang, Y. L., Li, L. C., Chen, W. Q., Xu, Z. H., et al. (2005). Histone acetylation affects expression of cellular patterning genes in the Arabidopsis root epidermis. Proc. Natl. Acad. Sci. U.S.A. 102, 14469-14474. doi: 10.1073/pnas.0503143102

Yang, J. Y., Iwasaki, M., Machida, C., Machida, Y., Zhou, X. P., and Chua, N. H. (2008). beta C1, the pathogenicity factor of TYLCCNV, interacts with AS1 to alter leaf development and suppress selective jasmonic acid responses. Genes Dev. 22, 2564-2577. doi: 10.1101/gad.1682208

Yang, X. J., and Seto, E. (2007). HATs and HDACs: from structure, function and regulation to novel strategies for therapy and prevention. Oncogene 26, 5310-5318. doi: 10.1038/sj.onc.1210599

Yoo, S. D., Cho, Y. H., and Sheen, J. (2007). Arabidopsis mesophyll protoplasts: a versatile cell system for transient gene expression analysis. Nat. Protoc. 2, 1565-1572. doi: 10.1038/nprot.2007.199

Yu, C. W., Liu, X. C., Luo, M., Chen, C. Y., Lin, X. D., Tian, G., et al. (2011). HISTONE DEACETYLASE6 interacts with FLOWERING LOCUS D and regulates flowering in Arabidopsis. Plant Physiol. 156, 173-184. doi: 10.1104/pp.111.174417

Zhou, C. H., Labbe, H., Sridha, S., Wang, L., Tian, L., Latoszek-Green, M., et al. (2004). Expression and function of HD2-type histone deacetylases in Arabidopsis development. Plant J. 38, 715-724. doi: 10.1111/j.1365313X.2004.02083.x

Zhou, Y., Tan, B., Luo, M., Li, Y., Liu, C., Chen, C., et al. (2013). HISTONE DEACETYLASE19 interacts with HSL1 and participates in the repression of seed maturation genes in Arabidopsis seedlings. Plant Cell 25, 134-148. doi: 10.1105/tpc.112.096313

Conflict of Interest Statement: The authors declare that the research was conducted in the absence of any commercial or financial relationships that could be construed as a potential conflict of interest.

Received: 23 September 2014; accepted: 10 December 2014; published online: 06 January 2015.

Citation: Zhao L, Lu J, Zhang J, Wu P-Y, Yang S and Wu K (2015) Identification and characterization of histone deacetylases in tomato (Solanum lycopersicum). Front. Plant Sci. 5:760. doi: 10.3389/fpls.2014.00760

This article was submitted to Plant Genetics and Genomics, a section of the journal Frontiers in Plant Science.

Copyright (C) 2015 Zhao, Lu, Zhang, Wu, Yang and Wu. This is an open-access article distributed under the terms of the Creative Commons Attribution License (CC BY). The use, distribution or reproduction in other forums is permitted, provided the original author(s) or licensor are credited and that the original publication in this journal is cited, in accordance with accepted academic practice. No use, distribution or reproduction is permitted which does not comply with these terms. 